R. Willstätter und H. H. Escher: Uber den Farbstoff der Tomate. (Zeitschr. physiol. Chem, 1910, 64, 47-61.) - Der "Lycopin" genannte Farbstoff der Tomate wurde von Montanari für ein Kondensationsprodukt aus zwei Molekülen Carotin gehalten. Da die von Arnaud aufgestellte Formel des Carotins vor kurzem erst durch Willstäter und Mieg $(\mathbf{Z} .1909,17,464)$ berichtigt worden ist, so haben die Verff, das Lycopin aufs neue einer Untersuchung unterworfen. Lycopin und Carotin unterscheiden sich ganz charakteristisch voneinander. Die Analyse des Lycopins stimmt für das Atomverhältnis des Carotins; die Molekulargewichte sind auf Grund von ebullioskopischen Bestimmungen in verschiedenen Lösungsmitteln übereinstimmend $=536$. Lycopin und Carotin sind also isomer gemäb der Formel $\mathrm{C}_{40} \mathrm{H}_{56}$. Trotzdem ist der Tomatenfarbstoff $\nabla$ om Carotin in seinen Eigenschaften, namentlich in der Form und Farbe der Krystalle und in der Farbe der Lösungen, auffällig verschieden, worüber nähere Mitteilungen gemacht werden. Ferner liefern die Verff. weitere ergänzende Angaben zur Kenntnis des Carotins: die Autooxydation und das Verhalten des Carotins gegen Halogene. Ein dem Bromid des Carotins $\left(\mathrm{C}_{40} \mathrm{H}_{36} \mathrm{Br}_{22}\right)$ ganz ähnliches sauerstofffreies Bromid wurde auch aus dem nach Willstätter und $\mathrm{Mieg}$ aus grünen Blättern isolierten Xanthophyll erhalten.

G. Sonntag.

M. Löwy: Der Champignon, eine indolbildende Pflanze. (Chem.Ztg. 1910, 34, 340.) - Verf, hat in den Champignonextrakten Indican nachgewiesen. Setzt man zu dem wässerigen oder alkoholischen Extrakte einige Tropfen konc. Salzsäure und dazu vorsichtig konc. Schwefelsäure, so färbt sich die ganze Lösung tiefblau. Braungefärbte Extraktlösungen dürfen nicht durch Tierkohle entfärbt werden.

J. Clement.

Th. B. Osborne und S. H. Clapp: Hydrolyse des krystallinischen Globulins des Kàrbissamens (Cucurbita maxima). (Zeitschr. analyt. Chem. 1910, 49, 146 bis 152.) - Vergl. Z, 1909, 18, 668.

\title{
Literatur.
}

Dr. phil. Konrad Bartelt: Die Terpene und Campherarten. Gr. $8^{\circ}$, VIII und 392 Seiten. Heidelberg 1908. Carl Winter's Universitätsbuchhandlung. - Das Gebiet der Terpene und der ihnen nahestehenden Körper war bis Mitte des verflossenen Jahrhunderts ein ziemlich dunkles geblieben. Seitdem ist es den Forschungen mehrerer Fachgenossen und einer mit steigendem Forschungserfolge zunehmenden Zahl von Chemikem gelungen, in das bis dahin regellos gehäufte Material von Tatsachen Ordnung und System za bringen, zahlreiche Konstitutionsformeln festzustellen und Darstellungsmethoden für die einzelnen Glieder der so interessanten und auch praktisch wichtigen Klasse von Körpern auszuarbeiten. - Unter den Meistern ant dem Felde der Terpen-Chemie seien die Namen Wallach, Semmler, Bertra $m$, Gildemeister hervorgehoben, und auch der Verfasser dieses Werkes hat sich experimentell mit der Materie befaßt, sodaß er berufen erscheint, uns eine Übersicht über das ganze Gebiet zu geben. Wir finden in dem seinem Lehrer Fr. W. Sem $\mathrm{mler}$ gewidmeten Werk die Hanpteinteilung in Kohlenwasseretoffe, Alkohole, Aldehyde, Ketone und Oxyde. Als Unterabteilungen figurieren in jeder Klasse die Grappen: Methanderivate, hydriert-cyelische Terpene, Sesquiterpene, Di- und Polyterpene. Weitere Trennungen erfolgen nach der Zahl der Ringe in mono-, bi- und tricyclische Gebilde, sodaf in dieses im einzelnen noch spezieller elweiterte Gebäude die bekannten und erforschten Repräsentanten zweckentsprechend untergebracht sind. Auf diese Weise ist in der Tat eine gate Utbersicht über das grofe Heer der T'erpenderivate gegeben, welche den Zweck der Orientierung und der Auffindung der einzelnen Glieder ermöglicht. Zahlreiche Konstitutionsformeln erleichtern das Verständnis der Körper und ihrer Unterschiede. Ausführliche Literatur-Angaben weisen dem Leser den Weg zur Vertiefung des Studiums.

G. Kassner.

Dr. Max Scholtz, a. o. Professor der pharmazeutischen Chemie an der Universität Greifswald: Lehrbuch der pharmazeutischen Chemie, I. Band. Anorganischer Teil. 
Gr. $8^{\circ} . \mathrm{X}$ und 488 Seiten. Mit 56 Abbildongen und einer Spektraltafel. Heidelberg 1910. CarI Winter's Universitätsbuchhandlung. Preis 10,60 Mk., geb. $12 \mathrm{Mk}$. - Das auf der Basis der modernen chemischen Auffassung des gesamten Tatsachenmaterials geschriebene Werk kann als ein recht gutes Lehrbuch der Chemie für Pharmazeuten betrachtet werden. Es vermittelt in nicht zu weitem Rahmen und in gut verständlicher Sprache die Kenntnis der die Materie beherrschenden Fundamentalgesetze. Es bringt die Konstitutionsformeln der wichtigsten Verbindungen, einen großen Tell der Umsetzungsformeln vom Standpunkte der Energetik bezw. elektrischer Ionen-Theorie, zeigt an vielen Stellen, wie sich die einzelnen Erscheinungen als Spezialfälle den allgemeinen Gesetzen unterordnen und teilt neben der Beschreibung der einzelnen Verbindungen auch Darstellungs- und Prüfungsvorschriften mit, letztere vorzugsweise bei den arzneilich angewendeten Substanzen. Bei den Metallen sind die für die Analyse wichtigen Umsetzungen jedesmal am Schlusse der einzelnen Kapitel übersichtlich zusammengestellt. Das Bestreben des Verf.'s beim Unterricht und beim Studium der Chemie mehr das Verständnis für die Erscheinungen zu wecken als das Gedächtnis durch Anfnahme eines umfangreichen Tatsachenmaterials übermäßrig zu belasten, scheint mir durch die Anordnung des Stoffes und seine Durchdringung mit den die Materie beherrsehenden Gesetzen in befriedigender Weise durch. geführt zu sein.

G. Kassner.

Dr. Ernst Winterstein, Professor, und Dr. Georg Trier, Assistent, an der Eidgenössischen Schule in Zürich: Die Alkaloid e. Eine Monographie der natürlichen Basen. Gr. $8^{\circ}$, VII und 340 Seiten. Berlin 1910. Verlag von Gebrüder Bornträger. Preis 11 Mk. Das vorliegende Buch ist ein Versuch, unsere derzeitige Kenntnis über die Alkaloide dem Leser in gedrängter Form zu vermitteln, wobei auch den physiologischen Eigenschaften der Pflanzenbasen besondere Berücksichtigung zuteil wurde. Während sich der erste Teil des aus 3 Hauptabschnitten sich zusammensetzenden Werkes mit den allgemeinen Eigenschaften der Alkaloide befafit (z. B. Begriff, Vorkommen, Darstellung, Konstitution, Erkennung. physiologisches Verhalten, Einteilung etc.), behandelt der $z$ weite umfangreiche Teil die einzelnen Basen in der Reihenfolge und inverhalb ibrer natürlichen Gruppen (z. B. Gruppe der aliphatischen Basen, Pyrrol-Gruppe, Pyridin-Gruppe, Chinolin-Gruppe u. s. w.). Der dritte Teil bespricht die Bedeutung der Alkaloide und ihre Entstehung in den Pflanzen. Es kann das Urteil über die uns von den Verfassern geschenkte Monographie dahin ausgesprochen werden, daß̧ sie uns eine recht gute Übersicht über das sehr umfangreicho Gebiet der Pflanzenbasen $\mathrm{zu}$ geben vermag, bei welcher auch jüngere Arbeiten über Konstitutionsfragen und Synthesen berücksichtigt sind. Ein klarer, deutlicher Druck, Veranschaulichung der Atomverkettungen durch zahlreiche, genaue Formelbilder sowie Angabe besonders ausgewählter Literatur-Zitate nnterstïtzen die Erreichung des beabsichtigten Zweckes. Bei der Aufzählung der AlkaloidReagenzien vermisse ich die Erwähnung der für manche Fälle wichtigen Selenschwefelsäure. Doch beeinträchtigen solche kleine Mängel den Wert des Buches nicht.

G. Kassner.

\section{Berichte ïber die Tätigkeit von Untersuchungsämtern etc.}

Jahresbericht des Chemischen Untersuchnngsamtes der Stadt Breslan für die Zeit vom 1. April 1909 bis 31. März 1910. Im Auftrage des Kuratoriums erstattet von Dr. H. Lührig, Direktor des Chemischen Untersuchungsamtes. Sonderabdruck aus Band 30, Heft I der "Breslauer Statistik". 50 S. Gr. $8^{\circ}$. Die Zahl der ausgeführten Untersuchungen betrug 16214, von denen 1948 vom Kgl. Polizeipräsidium, 472 von Gerichten und anderen Be hörden, 295 vom Magistrat, 176 von Privaten, 145 von der Auslandsfleischbeschaustelle, 1496 von der Kgl. Regierung, 11512 vom Wasserwerk und 170 vom Amte selbst veranlaßit waren. Es wurden u. a. untersucht: 802 Fleisch, 542 Wurst, 976 Milch, 51 Magermilch, 2 Buttermilch, 42 Rahm, 11 Ziegenmilch, 21 Käse, 449 Butter, 71 Margarine, 99 Schweinefett, 4 Kunstspeisefett, 34 Öle, 288 Müllereierzeugnisse, Back- und Teigwaren, 177 Gewürze, 125 Essig, 65 Zuckerwaren, 61 Fruchtsäfte und Obsterzeugnisse, 84 Frucht- und Gemüsedauerwaren, 50 Limonaden u. s. w., 59 Honig, 75 Spirituosen, 85 Wasser, 31 Hefe, 4 Bier, 245 Wein, 44 Kaffee und Ersatzstoffe, 57 Kakaowaren, 8 Tee, 18 Tabak, 61 Gebrauchsgegenstände u. s. w. - Fleisch: 3 Proben geräuchertes Schweinefieisch enthielten erhebliche Mengen Borsäure. Einige Hackfleischproben enthielten Schwefeldioxyd bis $0,179 \%$. - Wurst: Zahlreiche Verfälschungen mit stärkehaltigen Bındemitteln wurden festgestellt. Eine Mortadellawurst enthielt $0,093 \%$ Borsäure. Rotfärbung der Hällen kam öfters vor. Braunschweiger Mettwurst war mit würzelosem Paprika gefärbt. - Milch: Auffallend grof ist die Zabl der Beanstandungen wegen Wasserzusatzes. - Butter: Der Wassergehait stieg bis $49,26 \%$, der Fettgehalt sank bis $46,05 \%$. Der höchste Salzgehalt war 11,13\%. - Müllereierzeugnisse: Beanstandung fand künstliche Bleichung von Mehl. - Gew ürze: Safran mit 38,6\% Asche war mit Nitraten 